méditerranéenne, feuille 1: Mediterranée orientale. Editions du CNRS. Paris. 69p.

STRID, A. \& K. TAN -1997- Flora Hellenica. Koeltz Scientific Books, Konigstein.

TURLAND, N.J., L. CHILTON \& J.R. PRESS 1993- The flora of the Cretan area: annotated checklist \& Atlas. The Natural History Museum and HMSO, London.

ZANGHERI, P. - 1976- Flora italica. Vol.1. CedamPadova. p 1157.

ZOHARY, M. -1962- Plant life of Palesine. Ed. Ronald Press. p. 262.

ZOHARY, M. -1966- Flora palaestina. Vol. 1. The Israel academy of sciences and humanities, Goldberg's Press. p.364.
ZOHARY, M. -1973- The geobotanical foundations of the Middle East. Ed. Gustav Fisher. Stuttgart. p.738.

Aceptado para su publicación en septiembre de 2002

Dirección de los autores. J.M. Dufour-Dror: Geography department, Tel-Aviv University. P.O.B. 39040, Ramat-Aviv, Tel-Aviv 69978, Israel; Centre Biogéographie-Ecologie, Institut Européen pour le Développement Durable, 45 rue de France, 77300 Fontainebleau, France; A. Ertas: Department of Sylviculture, Faculty of Forestry, Istanbul University, 80895 Bahçekoy-Istanbul, Turkey.

\title{
109. NUEVAS LOCALIDADES Y UNA NUEVA VARIEDAD DE CENTAUREA ULTREIAE SILVA PANDO (COMPOSITAE).
}

New localities and a new variety of Centaurea ultreiae Silva Pando (Compositae).

Palabras clave. Centaurea, Compositae, corología, A Coruña, España.

Key Words. Centaurea, Compositae, corology, A Coruña, Spain.

Centaurea ultreiae Silva Pando, especie encuadrada en la categoría En Peligro (EN) (UICN, 2001), es conocida hasta el momento solamente de la localidad de donde fue descrita (A Coruña, Coristanco, Pena Cabaleira) situada en el macizo gábrico de los Montes do Castelo (Silva Pando,1987). La realización de trabajos forestales en esta zona durante estos cinco últimos años nos ha permitido ampliar el área de distribución de la especie con el hallazgo de diez nuevas poblaciones de este taxón en otros tres términos municipales, confirmar la estabilidad y relativa frecuencia de una variedad que describimos en esta nota y, por último, conocer el efecto sobre la localidad clásica de las obras de instalación de un parque eólico en la zona.

\section{Material estudiado}

Centaurea ultreiae Silva Pando in Anales Jard. Bot. Madrid 44: 422 (1987) 

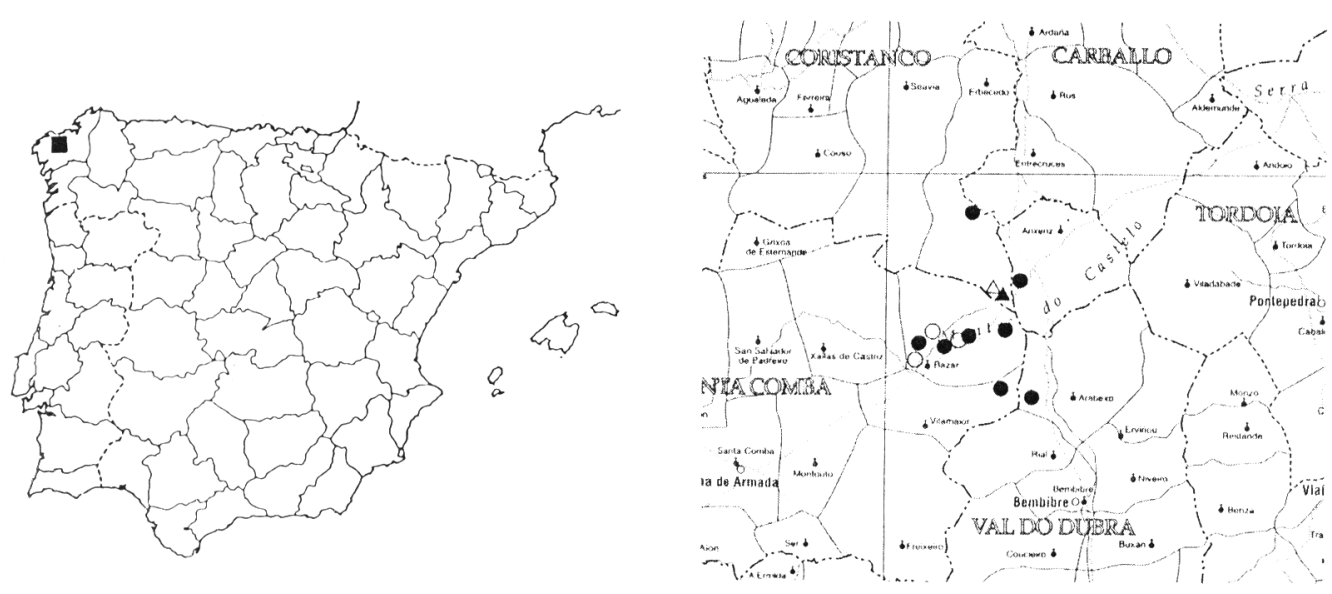

Figura 1: Distribución de Centaurea ultreiae (escala 1:200.000): (A) localidad clásica (var. bazarensis $(\Delta)$; $(\bigcirc)$ nuevas localidades: $(\bullet)$ :variedad típica;(O): var. bazarensis. Distribution of Centaurea ultreiae (climbs 1:200.000): (\) classic locality (var. bazarensis $(\Delta)$ ); $(\bigcirc)$ new localities: $(\bullet)$ typical variety; (O): var. bazarensis.

A CORUÑA: Coristanco, Erbecedo, 29TNH2474, 400 m., márgenes removidos de campo de fútbol y claros de bosque de Pinus radiata y Acacia melanoxylon, 11-VI-2002, J.B. Blanco-Dios, LOU 24808; Santa Comba, Bazar, 29TNH2269, $400 \mathrm{~m}$., taludes y cunetas en margen de bosque mixto de Pinus pinaster y Eucalyptus globulus, 11-VI-2002, J.B. Blanco-Dios, LOU 24809; idem, 29TNH2369,29TNH2469, 415-450 m, taludes y cunetas en margen de bosques de Eucalyptus globulus, 20-V-2002, 4-VI-2002, J.B. BlancoDios, LOU 24801, LOU 24802, LOU 24803, LOU 24804; idem, 29TNH2470, 29TNH2570, 490-500 m, taludes, cunetas e interior de reciente plantación de E. nitens, 20-V-2002, J.B. Blanco-Dios, LOU 24800; Santa Comba, Vilamaior, 29TNH2567, 29TNH2568, 430-450 $\mathrm{m}$, márgenes de bosques adultos e interior de plantaciones jóvenes de E. globulus, 4-VI2002, J.B. Blanco-Dios, LOU 24806; Tordoia, Anxeriz, 29TNH2571, 480-510 m, taludes y cunetas en margen de tojal-brezal, 20-V-2002,
J.B. Blanco-Dios, LOU 24807; Val do Dubra, Arabexo, 29TNH2667, 430-435 m, márgenes e interior de plantaciones jóvenes de E. globulus, 4-VI-2002, J.B. Blanco-Dios, LOU 24805.

Estas nuevas poblaciones también se asientan sobre suelos derivados de gabros (salvo la localizada en Santa Comba, Bazar (LOU 24809), sobre anfibolitas) y se adaptan a distintas exposiciones (taludes, cunetas, claros de plantaciones de diferentes edades de distintas especies exóticas...), mientras que en la localidad clásica coloniza caminos y cunetas. En las jóvenes plantaciones de Eucalyptus donde la hemos detectado observamos su tolerancia al laboreo del terreno (subsolado y fresado) y a la sombra de estas especies arbóreas, muy disminuida en la actualidad a causa de los serios problemas de defoliación que padecen por la incidencia de varias patologías (gorgojo (Gonipterus scutellatus Gyll.) y varios hongos del género Mycosphaerella), lo que hace que la luz llegue con facilidad al suelo de estas masas forestales. 
Otra consideración a realizar es que las nuevas poblaciones detectadas presentan mayoritariamente corolas amarillas, tal y como se especifica en la diagnosis original de esta especie, salvo en las poblaciones de Santa Comba, Bazar, donde hemos hallado varios grupos de plantas con corolas de color entre naranja, rosado y púrpura (LOU 24801, LOU 24802, LOU 24804). Esta característica ya se había observado en una parte de los especímenes de la localidad clásica (García Martínez et al. 1991), y hemos podido comprobar durante estos últimos años que la misma se mantiene hasta la actualidad tanto en estado silvestre como en cultivo. Por ello, hemos considerado conveniente describir esta diferencia respecto a la descripción original, por lo que proponemos la siguiente nueva variedad:

Centaurea ultreiae var. bazarensis BlancoDios, var. nov.

Typicae varietati valde similis, a qua differt corollis aurantiaco-roseis vel purpureoaurantiacis, nec luteis.

Etimología: recibe su nombre en honor a Bazar, parroquia del término municipal de Santa Comba en la que se localizan la mayor parte de sus poblaciones conocidas.

Holotypus. A CORUÑA: Santa Comba, Bazar, 29TNH2369, 440 m, taludes y cunetas en márgenes de bosque de Eucalyptus globulus, 20-V-2002, J.B. Blanco-Dios, LOU 24801.

Con estas nuevas poblaciones contribuimos a aumentar las existencias de esta especie en unas 2000 plantas, oscilando entre 25 y 1000 el número de especímenes contabilizados por población. De la mentada cantidad total, unas 150 corresponderían a la nueva variedad, que convive con la variedad tipo en cuatro de las once poblaciones conocidas de esta especie. Fig. 1.
AGRADECIMIENTOS. Al Dr. F.J. Silva Pando, conservador del herbario LOU, por las sugerencias aportadas al texto original; a J. Gómez y M. J. Sánchez por su agradable compañía y su paciencia.

\section{BIBLIOGRAFÍA}

GARCÍA MARTÍNEZ, X.R., E. VALDÉSBERMEJO, F.J. SILVA PANDO, V. RODRIGUEZ GRACIA, \& , F. GÓMEZ VIGIDE -1991-. Aportaciones a la Flora de Galicia, IV. Nova Acta Científica Compostelana (Bioloxía), 2: 41-56.

SILVA PANDO, F.J. - 1987-. Centaurea ultreiae Silva Pando, sp. nov. (Compositae) del noroeste de España. Anales Jard. Bot. Madrid 44 (2): 421-427.

UICN -2001-. Categorias y Criterios de la Lista Roja de la UICN: Versión 3.I. Comisión de Supervivencia de Especies de la UICN. UICN, Gland, Suiza y Cambridge, Reino Unido. ii+33 pp.

Aceptado para su publicación en septiembre de 2002

Dirección del autor. Escola de Capacitación e Experimentación Agraria de Lourizán. Apdo 127. 36080- Pontevedra. 